\title{
The optimal control of storage for arbitrage and buffering, with energy applications
}

\author{
James Cruise* and Stan Zachary*
}

March 1, 2018

\begin{abstract}
We study the optimal control of storage which is used for both arbitrage and buffering against unexpected events, with particular applications to the control of energy systems in a stochastic and typically time-heterogeneous environment. Our philosophy is that of viewing the problem as being formally one of stochastic dynamic programming, but of using coupling arguments to provide good estimates of the costs of failing to provide necessary levels of buffering. The problem of control then reduces to that of the solution, dynamically in time, of a deterministic optimisation problem which must be periodically re-solved. We show that the optimal control then proceeds locally in time, in the sense that the optimal decision at each time $t$ depends only on a knowledge of the future costs and stochastic evolution of the system for a time horizon which typically extends only a little way beyond $t$. The approach is thus both computationally tractable and suitable for the management of systems over indefinitely extended periods of time. We develop also the associated strong Lagrangian theory (which may be used to assist in the optimal dimensioning of storage), and we provide characterisations of optimal control policies. We give examples based on Great Britain electricity price data.
\end{abstract}

\section{Introduction}

The control of complex stochastic systems, for example modern power networks which must cope with many sources of uncertainty in both generation and demand, requires real-time optimisation of decision problems which are often computationally intractablenotably so in a time-heterogeneous environment. This clearly also poses difficulties for the design of such systems. As in the case of the well studied areas of communication and manufacturing networks, our belief is that what is required is the careful specification of the stochastic models governing the behaviour of such systems, coupled with the analytical derivation of accurate approximation techniques.

In the present paper we use an economic framework to consider the optimal control of a single storage facility. The problem is made interesting because, at least in power networks, storage may be simultaneously used for many different purposes, with potentially conflicting objective functions. However, if storage is to be economically viable, it must be capable of meeting these competing objectives. We concentrate on energy storage in a time-heterogeneous environment, and consider two of the main uses of such storage systems: (a) price arbitrage, i.e. the buying and selling of energy over time (whether to

${ }^{*}$ Heriot-Watt University. Research supported by EPSRC grant EP/I017054/1 
earn revenue for the store owner or for the benefit of the consumer), and (b) the provision of buffering services, so as to react rapidly to sudden and unexpected changes, for example the loss of a generator or transmission line, or a sudden surge in demand. Our general approach is likely to be applicable to other uses of storage, and also to the optimal control of other facilities used for the provision of multiple services.

There is considerable literature on the control of storage for each of the above two purposes considered on its own. In the case of the use of storage for arbitrage, and with linear cost functions for buying and selling at each instant in time, the problem of optimal control is the classical warehouse problem (see [1, 2, 3] and also [4] for a more recent example). Cruise et al [5] consider the optimal control of storage - in both a deterministic and a stochastic setting - in the case where the store is a price maker (i.e. the size of the store is sufficiently large that its activities influence prices in the market in which it operates) and is subject to both capacity and rate constraints; they develop the associated Lagrangian theory, and further show that the optimal control at any point in time usually depends only on the cost functions associated with a short future time horizon. Recent alternative approaches for studying the value and use of storage for arbitrage can be found in the papers $[6,7,8,9,10]$ — see also the text [11], and the further references given in [5]. For an assessment of the potential value of energy storage in the UK electricity system see [12].

There have been numerous studies into the use of storage for buffering against both the increased variability and the increased uncertainty in electrical power systems, due to higher penetration of renewable penetration - the former due to the natural variability of such resources as wind power, and the latter due to the inherent uncertainty of forecasting. These studies have considered many different more detailed objectives; these range from the sizing and control of storage facilities co-located with the renewable generation so as to provide a smoother supply and so offset the need for network reinforcement $[13,14,15]$, to studies on storage embedded within transmission networks so as to increase wind power utilisation and so reduce overall generation costs $[16,17,18]$. In addition there have been a number of studies into the more general use of storage for buffering, for example, so as to provide fast frequency response to power networks $[19,20,12]$, or to provide quality of service as part of a microgrid [21, 22].

In general the problem of using a store for buffering is necessarily stochastic. The natural mathematical approach is via stochastic dynamic programming. This, however, is liable to be computationally intractable, especially in the case of long time horizons and the likely time heterogeneity of the stochastic processes involved. Therefore much of the literature considers necessarily somewhat heuristic but nevertheless plausible control policies - again often adapted to meeting a wide variety of objectives. For example, for storage embedded in a distribution network, two control policies are considered in [23]; the first policy aims to feed into a store only when necessary to keep local voltage levels within a predefined range and to empty the store again as soon as possible thereafter; the second policy aims to maintain a constant level of load in the network. For larger stores operating within transmission networks, the buffering policies studied have included that of a fixed target level policy [24], a dynamic target level policy [25], and a two stage process with day ahead generation scheduling and a online procedure to adapt load levels [26].

Control policies have been studied via a range analytic and simulation based methods. Examples of an analytic approach can be found in [27], where partial differential equations are utilised to model the behaviour and control of a store, and in [23, 28], where spectral analysis of wind and load data is used with models which also incorporate tur- 
bine behaviour. Simulation-based studies include [24, 25], which use a bootstrap approach based on real wind forecast error data, and [26], which uses Monte Carlo simulation of the network state.

In the present paper we study the optimal control of a store which is used both for arbitrage and for buffering against unpredictable events. As previously indicated we use an economic framework, so that the store sees costs (positive or negative) associated with buying and selling, and with the provision of buffering services. The store seeks to operate in such a way as to minimise over time the sum of these costs. We believe such an economic framework to be natural when the store operates as part of some larger and perhaps very complex system, provided the price signals under which the store operates are correctly chosen. The store may be sufficiently large as to have market impact, leading to nonlinear cost functions for buying and selling, may be subject to rate (as well as capacity) constraints, and, as will typically be the case, may suffer from round-trip inefficiencies. We formulate a stochastic model which is realistic in many circumstances and characterise some of the properties of an optimal control, relating the results to the existing experimental literature. We develop the associated strong Lagrangian theory and, by making a modest approximation - the validity of which may be tested in practical applications show how to construct a computationally tractable optimal control. These latter results form a nontrivial extension of those of the "arbitrage-only" case studied in [5], and require significant new developments of the necessary optimization theory; as in [5], the optimal control at any time usually depends on a relatively short time horizon (though one which is typically somewhat longer than in the earlier case), so that the algorithm is suitable for the optimal control of the store over an indefinite period of time.

The optimal control is given by the solution, at the start of the control period, of a deterministic optimisation problem which can be regarded as that of minimising the costs associated with the store buying and selling added to those of notionally "insuring" for each future instant in time against effects of the random fluctuations resulting from the provision of buffering services. The cost of such "insurance" depends on the absolute level of the store at that time. Thus this deterministic problem is that of choosing the vector of successive levels of the store so as to minimise a cost function $\sum_{t}\left[C_{t}\left(x_{t}\right)+A_{t}\left(s_{t}\right)\right]$, subject to rate and capacity constraints, where $C_{t}\left(x_{t}\right)$ is the cost of incrementing the level of the store (positively or negatively) at time $t$ by $x_{t}$, and the "penalty" function $A_{t}$ is such that $A_{t}\left(s_{t}\right)$ is the expected cost of any failure to provide the required buffering services at the time $t$ when the level of the store is then $s_{t}$. We define this optimisation problem $\mathbf{P}$ more carefully in Sections 2 and 5. In the stochastic environment in which the store operates, the solution of this deterministic problem determines the future control of the store until such time as its buffering services are actually required, following which the level of the store is perturbed and the optimisation problem must be re-solved starting at the new level. The continuation of this process provides what is in principle the exactly optimal stochastic control of the store on a potentially indefinite time scale.

In Section 2 we formulate the relevant stochastic model and discuss its applicability. This enables us, in Section 3 to provide some characteristic properties of optimal solutions, which we relate to empirical work in the existing literature. In Sections 4 and 5 we develop the approach to an optimal control outlined above. Section 6 considers the deterministic optimisation problem associated with the stochastic control problem and derives the associated strong Lagrangian theory, while in Section 7 we develop an efficient algorithm. Section 8 gives examples. 


\section{Problem formulation}

Consider the management of a store over a finite time interval $[0, T]$ where the time horizon $T$ is integer, and where $[0, T]$ is divided into a succession of periods $t=1, \ldots, T$ of integer length. At the start of each time period $t$ the store makes a decision as to how much to buy or sell during that time period; however, the level of the store at the end of that time period may be different from that planned if, during the course of the period, the store is called upon to provide buffering services to deal with some unexpected problem or shock. Such a shock might be the need to supply additional energy during the time period $t$ due to some unexpected failure - for example that of a generator - or might simply be the difference between forecast and actual renewable generation or demand. We suppose that the capacity of the store during the time period $t$ is $E_{t}$ units of energy. (Usually $E_{t}$ will be constant over time, but need not be, and there are some advantages - see in particular Section 4-in allowing the time dependence.) Similarly we suppose that the total energy which may be input or output during the time period $t$ is subject to rate (i.e. power) constraints $P_{I t}$ and $P_{O t}$ respectively. This slotted-time model corresponds, for example, to real world energy markets where energy is typically traded at half-hourly or hourly intervals, with the actual delivery of that energy occurring in the intervening continuous time periods. Detailed descriptions of the operation of the UK market can be found in $[29,30]$.

For each $t$ let $X_{t}=\left\{x:-P_{O t} \leq x \leq P_{I t}\right\}$. Both buying and selling prices associated with any time period $t$ may be represented by a convex function $C_{t}$ defined on $X_{t}$ which is such that, for positive $x, C_{t}(x)$ is the price of buying $x$ units of energy for delivery during the time period $t$, while, for negative $x, C_{t}(x)$ is the negative of the price for selling $-x$ units of energy during that time period. Thus, in either case, $C_{t}(x)$ is the cost of a planned change of $x$ to the level of the store during the time period $t$, in the absence of any buffering services being required during the course of that time period. The convexity assumption corresponds, for each time $t$, to an increasing cost to the store of buying each additional unit, a decreasing revenue obtained for selling each additional unit, and every unit buying price being at least as great as every unit selling price. When, as is usually the case, the store is not perfectly efficient in the sense that only a fraction $\eta \leq 1$ of the energy input in available for output, then this may be captured in the cost function by reducing selling prices by the factor $\eta$; under the additional assumption that the cost functions $C_{t}$ are increasing it is easily verified that this adjustment preserves the above convexity of the functions $C_{t}$. We thus assume that the cost functions are so adjusted so as to capture any such round-trip inefficiency.

Remark 1. A further form of possible inefficiency of a store is leakage, whereby a fraction of the contents of the store is lost in each unit of time. We do not explicitly model this here. However, only routine modifications are required to do so, and are entirely analogous to those described in [5].

Remark 2. Note also that, in the above model, it is possible to absorb the rate constraints into the cost functions - by setting the costs associated with $x \notin X_{t}$ to be prohibitively high - and to preserve the convexity of these functions. However, in general we prefer to avoid this approach here.

Suppose that at the end of the time period $t-1$, or equivalently at the start of the time period $t$, the level of the store is $s_{t-1}$ (where we take $s_{0}$ to be the initial level of the store). We assume that one may then choose a planned adjustment (contract to buy or 
sell) $x_{t} \in X_{t}$-and such that additionally $s_{t-1}+x_{t} \in\left[0, E_{t}\right]$ - to the level of the store during the time period $t$, the cost of this adjustment being $C_{t}\left(x_{t}\right)$. Subsequent to this, during the course of the time period $t$, the the store may subject to some shock or random disturbance, corresponding perhaps to the need to provide unexpected buffering services, which may both disturb the final level of the store at the end of that time period - and perhaps also at the end of subsequent time periods - and have further associated costs, the latter being typically those of the store not being able to provide the required services. For each $t$, and for each possible level $s_{t-1}$ of the store at the end of the time period $t-1$, define $V_{t-1}\left(s_{t-1}\right)$ to be the expected future cost of subsequently managing the store under an optimal strategy (i.e. one under which this expected cost is minimised), under the assumption that either no shocks have occurred by the end of the time period $t-1$ or that, given the level $s_{t-1}$, such past shocks as have occurred by that time do not influence the optimal future management of the store or its associated costs. Under these conditions, and for a planned adjustment $x_{t}$ to the level of the store during the time period $t$ (at an immediate cost $C_{t}\left(x_{t}\right)$ as indicated above), in the absence of any shock during the time period $t$, the expected cost of optimally managing the store thereafter is then $V_{t}\left(s_{t-1}+x_{t}\right)$. We assume that the expected additional cost to the store, both immediate and future, of dealing optimally with any shock which may occur during the time period $t$ is a function $A_{t}\left(s_{t-1}+x_{t}\right)$ of the planned level $s_{t-1}+x_{t}$ of the store for the end of the time period $t$. We then have that

$$
V_{t-1}\left(s_{t-1}\right)=\min _{\substack{x_{t} \in X_{t} \\ s_{t-1}+x_{t} \in \cap\left[0, E_{t}\right]}}\left[C_{t}\left(x_{t}\right)+A_{t}\left(s_{t-1}+x_{t}\right)+V_{t}\left(s_{t-1}+x_{t}\right)\right]
$$

and that the optimal planned increment to the level of the store for the time period $t$ (given that an optimal policy is to be followed thereafter) is given by $\hat{x}_{t}\left(s_{t-1}\right)$ where this is defined to be the value of $x_{t} \in X_{t}$ which achieves the minimisation in the recursion (1).

We also define the terminal condition

$$
V_{T}\left(s_{T}\right)=0
$$

for all possible levels $s_{T}$ of the store at the end of the time period $T$.

Note that $A_{t}\left(s_{t-1}+x_{t}\right)$ (which may be alternatively be interpreted as the "insurance" cost associated with the planed level of the store for the time period $t$ as described in the Introduction) may be understood via a coupling argument, in which the possibly disturbed and subsequently optimally controlled process of store levels - following any shock in the time period $t$-is coupled to the process which is undisturbed in that time period and subsequently optimally controlled; $A_{t}\left(s_{t-1}+x_{t}\right)$ is then the expected difference in the costs of operating the two processes until such time (if ever) as they subsequently merge. As we discuss further below, this interpretation proves useful in finding workable approximations to the functions $A_{t}$.

Remark 3 . We make the assumption above that each function $A_{t}$, representing the extra cost of dealing with a shock occurring during the time period $t$, may be represented as a function of the planned level $s_{t-1}+x_{t}$ of the store for the end of that time period and, given this, does not further depend on the level $s_{t-1}$ of the store at the beginning of that time period. The accuracy of this assumption will vary according to the precise characteristics of the store, the way in which it interacts with its external environment in the event of shocks, and the various cost functions which form part of the model. The assumption is likely to be at its most accurate when rate constraints do not play a major role in the management 
of the store, as the store may adjust to its target levels quickly. Elsewhere, when the level of the store does not change too much during a single time period, the assumption may still be regarded as a reasonable approximation. Its relaxation - for example by allowing $A_{t}$ to be a more general function of $s_{t-1}$ and $x_{t}$-simply complicates without essentially changing the analysis below.

Our aim is to determine the optimal control of the store over the time interval $[0, T]$. Such a control will necessarily be stochastic. In principle some form of stochastic dynamic programming approach is required. However, particularly within a time heterogeneous environment (in which there may be no form of regularity in either the functions $C_{t}$ or in the shock processes), such an approach would be unlikely to be efficient, and might well prove computationally intractable, on account of (a) the need, in such an approach, to completely determine each of the functions $V_{t}$ defined above, and (b) the need to solve the problem over the entire time interval $[t, T]$ in order to determine the optimal control at any time $t$.

Our method of proceeding is therefore as follows. We assume that the functions $A_{t}$ are known, at least to within reasonable approximations. (We argue in Section 4 that in many cases the functions $A_{t}$ may be determined either exactly or to within a very good approximation; this follows from the coupling characterisation of these functions introduced above.) Given the initial level $s_{0}$ of the store we may then use the argument leading to the recursion (1) and (2) to determine very efficiently a control which remains optimal up to the end of the first time period in which a shock actually occurs. Following such a shock (and, if necessary, once its knock-on effects have cleared from the system-again see the discussion of Section 4), the current state (level) of the store is reexamined and the optimal future control strategy recalculated. Iteration of this process leads to an efficient (stochastic) dynamic control for the entire time interval $[0, T]$. We also show below that typically the optimal decision at (the start of) any time $t$ depends only on the functions $C_{t^{\prime}}$ and $A_{t^{\prime}}$ for values of time $t^{\prime}$ extending only a little beyond the time $t$. The approach outlined above is therefore generally also suitable for the ongoing optimal management of the store over an indefinite period of time.

\section{Characterisation of optimal solutions}

In this section we establish some properties of the functions $\hat{x}_{t}(\cdot)$ defined in the previous section and determining the optimal control of the store.

One case which will be of particular interest is that where the store is a price taker (i.e. the store is not so large as to impact itself on market prices), so that, for each $t$, the cost function $C_{t}$ is given by

$$
C_{t}(x)= \begin{cases}c_{t}^{(b)} x, & \text { if } x \geq 0 \\ c_{t}^{(s)} x, & \text { if } x<0\end{cases}
$$

and where the unit "buying" price $c_{t}^{(b)}$ and the unit "selling" price $c_{t}^{(s)}$ are such that $c_{t}^{(s)} \leq c_{t}^{(b)}$. (That, at any time $t$, the reward obtained in the market resulting from decreasing the level of the store by a single unit may be less than the cost of increasing the level of the store by a single unit may primarily reflect the fact that the store may be less than perfectly efficient - see the discussion of Section 2.)

Proposition 1 below is a very simple result which shows that in the case where buying and selling prices are equal (typically corresponding to a perfectly efficient store), and 
provided rate constraints are nonbinding, the optimal policy is a "target" one. By this we mean that for each time period $t$ there exists a target level $\hat{s}_{t}$ : given that the level of the store at the end of the immediately preceding time period is $s_{t-1}$ and that shocks prior to that time have no further ongoing effects on the management of the store, the optimal planned level $s_{t-1}+x_{t}$ of the store to be achieved during the time period $t$ is set to some value $\hat{s}_{t}$, independently of $s_{t-1}$.

Proposition 1. Suppose that, for each $t$, we have $c_{t}^{(b)}=c_{t}^{(s)}=c_{t}$ say; define

$$
\hat{s}_{t}=\underset{s \in\left[0, E_{t}\right]}{\arg \min }\left[c_{t} s+A_{t}(s)+V_{t}(s)\right] .
$$

Then, for each $t$ and for each $s_{t-1}$, we have $\hat{x}_{t}\left(s_{t-1}\right)=\hat{s}_{t}-s_{t-1}$ provided only that this quantity belongs to the set $X_{t}$.

Proof. The recursion (1) here becomes, for each $t$,

$$
V_{t-1}\left(s_{t-1}\right)=\min _{\substack{x_{t} \in X_{t} \\ s_{t-1}+x_{t} \in \cap\left[0, E_{t}\right]}}\left[c_{t} x_{t}+A_{t}\left(s_{t-1}+x_{t}\right)+V_{t}\left(s_{t-1}+x_{t}\right)\right]
$$

and the above minimisation is achieved by $x_{t}$ such that $s_{t-1}+x_{t}=\hat{s}_{t}$, provided only that $x_{t} \in X_{t}$.

In order to deal with the possibility of rate constraint violation, with the more general price-taker case where $c_{t}^{(s)}<c_{t}^{(b)}$, and with the quite general case where the cost functions $C_{t}$ are merely required to be convex, we require the additional assumption of convexity of the functions $A_{t}$. This latter condition, while not automatic, is reasonably natural in many applications - see the examples of Section 8.

Proposition 2. Suppose that, in addition to convexity of the functions $C_{t}$, each of the functions $A_{t}$ is convex. Then, for each $t$ :

(i) the function $V_{t-1}$ is convex;

(ii) $\hat{x}_{t}\left(s_{t-1}\right)$ is a decreasing function of $s_{t-1}$;

(iii) $s_{t-1}+\hat{x}_{t}\left(s_{t-1}\right)$ is an increasing function of $s_{t-1}$.

Proof. It is helpful to define, for each $t=1, \ldots, T$, the function $U_{t-1}$ of each possible level $s_{t-1}$ of the store at the end of the time period $t-1$, and each possible planned increment $x_{t}$ to the level of the store for the time period $t$ by

$$
U_{t-1}\left(s_{t-1}, x_{t}\right)=C_{t}\left(x_{t}\right)+A_{t}\left(s_{t-1}+x_{t}\right)+V_{t}\left(s_{t-1}+x_{t}\right) .
$$

The recursion (1) now becomes

$$
V_{t-1}\left(s_{t-1}\right)=\min _{\substack{x_{t} \in X_{t} \\ s_{t-1}+x_{t} \in \cap\left[0, E_{t}\right]}} U_{t-1}\left(s_{t-1}, x_{t}\right)
$$

To show (i) we use backwards induction in time. The function $V_{T}$ is convex. Suppose that, for any given $t \leq T$, the function $V_{t}$ is convex; we show that the function $V_{t-1}$ is convex. For each of given values $s_{t-1}^{(i)}, i=1, \ldots, n$ of $s_{t-1}$, let $x_{t}^{(i)}$ be the value of $x_{t}$ which achieves the minimisation in (7), and for any convex combination $\bar{s}_{t-1}=\sum_{i=1}^{n} \kappa_{i} s_{t-1}^{(i)}$, where each 
$\kappa_{i} \geq 0$ and where $\sum_{i=1}^{n} \kappa_{i}=1$, define also $\bar{x}_{t}=\sum_{i=1}^{n} \kappa_{i} x_{t}^{(i)}$. Note that $\bar{x}_{t} \in X_{t}$ and that $\bar{s}_{t-1}+\bar{x}_{t} \in\left[0, E_{t}\right]$. Then, from $(7)$,

$$
\begin{aligned}
V_{t-1}\left(\bar{s}_{t-1}\right) & \leq U_{t-1}\left(\bar{s}_{t-1}, \bar{x}_{t}\right) \\
& \leq \sum_{i=1}^{n} \kappa_{i} U_{t-1}\left(s_{t-1}^{(i)}, x_{t}^{(i)}\right) \\
& =\sum_{i=1}^{n} \kappa_{i} V_{t-1}\left(s_{t-1}^{(i)}\right),
\end{aligned}
$$

where the second line in the above display follows from the definition (6) of the function $U_{t-1}$ and the convexity of the functions $C_{t}, A_{t}$ and $V_{t}$ (the latter by the inductive hypothesis). Thus $V_{t-1}$ is convex as required.

To show (ii) and (iii), given values $s_{t-1}^{(1)} \leq s_{t-1}^{(2)}$ of $s_{t-1}$, again let $x_{t}^{(1)}, x_{t}^{(2)}$ be the respective values of $x_{t}$ which achieves the minimisation in (7). Since for the function $U_{t-1}\left(s_{t-1}^{(1)}, \cdot\right)$ is minimised in $X_{t} \cap E_{t}$ at $x_{t}^{(1)}$, it follows straightforwardly, from the definition (6) of the function $U_{t-1}$ and the convexity of the function $C_{t}$ and that of the function $A_{t}+V_{t}$, that, since $s_{t-1}^{(1)} \leq s_{t-1}^{(2)}$, the minimisation of the function $U_{t-1}\left(s_{t-1}^{(2)}, \cdot\right)$ is achieved (or, in the case of nonuniqueness, may be achieved) at $x_{t}^{(2)} \leq x_{t}^{(1)}$. Thus the result (ii) follows. Similarly, it is again straightforward from the convexity of the function $C_{t}$ and that of the function $A_{t}+V_{t}$ and since $s_{t-1}^{(1)} \leq s_{t-1}^{(2)}$, that $x_{t}^{(2)}$ is (or, in the case of nonuniqueness, may be taken to be) such that $s_{t-1}^{(2)}+x_{t}^{(2)} \geq s_{t-1}^{(1)}+x_{t}^{(1)}$. The result (iii) thus similarly follows.

Remark 4. Note that the rate constraints $x_{t} \in X_{t}$ for all $t$ cause no difficulties for the above proof - a result which may alternatively be seen by absorbing these constraints into the cost functions $C_{t}$ as described in Remark 2 .

We now return to the price-taker case, in which the cost functions are as defined by (3), and which corresponds to a store which is not sufficiently large as to have market impact. Here we may prove a strengthened version of Proposition 2. For each $t$, given that the function $A_{t}$ is convex, define

$$
s_{t}^{(b)}=\underset{s \in\left[0, E_{t}\right]}{\arg \min }\left[c_{t}^{(b)} s+A_{t}(s)+V_{t}(s)\right]
$$

and similarly define

$$
s_{t}^{(s)}=\underset{s \in\left[0, E_{t}\right]}{\arg \min }\left[c_{t}^{(s)} s+A_{t}(s)+V_{t}(s)\right] .
$$

Note that the above convexity assumption and the condition that, for each $t$, we have $c_{t}^{(s)} \leq c_{t}^{(b)}$ imply that $s_{t}^{(b)} \leq s_{t}^{(s)}$. We now have the following result.

Proposition 3. Suppose that the cost functions $C_{t}$ are as given by (3) and that the functions $A_{t}$ are convex. Then the optimal policy is given by: for each $t$ and given $s_{t-1}$, take

$$
x_{t}= \begin{cases}\min \left(s_{t}^{(b)}-s_{t-1}, P_{I t}\right) & \text { if } s_{t-1}<s_{t}^{(b)}, \\ 0 & \text { if } s_{t}^{(b)} \leq s_{t-1} \leq s_{t}^{(s)}, \\ \max \left(s_{t}^{(s)}-s_{t-1},-P_{O t}\right) & \text { if } s_{t-1}>s_{t}^{(s)} .\end{cases}
$$

Proof. For each $t$, it follows from the convexity of the functions $C_{t}, A_{t}$ and $V_{t}$ (the latter by the first part of Proposition 2) that, for $s_{t-1}<s_{t}^{(b)}$ the function $C_{t}\left(x_{t}\right)+A_{t}\left(s_{t-1}+\right.$ 
$\left.x_{t}\right)+V_{t}\left(s_{t-1}+x_{t}\right)$ is minimised by $x_{t}=s_{t}^{(b)}-s_{t-1}$, for $s_{t}^{(b)} \leq s_{t-1} \leq s_{t}^{(s)}$ it is minimised by $x_{t}=0$, while for $s_{t-1}>s_{t}^{(s)}$, it is minimised by $x_{t}=s_{t}^{(s)}-s_{t-1}$. The required result now follows from the recursion (1).

Thus in general in the price-taker case there exists, for each time period $t$, a "target interval" $\left[s_{t}^{(b)}, s_{t}^{(s)}\right]$ such that, if the level of the store at the end of the previous time period is $s_{t-1}$ (and again given that the shocks prior to this time have no ongoing effects on the optimal management of the store), the optimal policy is to chose $x_{t}$ so that $s_{t-1}+x_{t}$ is the nearest point (in absolute distance) to $s_{t-1}$ lying within, or as close as possible to, the above interval. In the case where $c_{t}^{(b)}=c_{t}^{(s)}=c_{t}$, the above interval shrinks to the single point $\hat{s}_{t}$ defined by (4).

These results shed some light on earlier, more applied, papers of Bejan et al [24] and Gast et al [25], in which the uncertainties in the operation of a energy store result from errors in wind power forecasts. The model considered in those papers is close to that of the present paper, as we now describe. The costs of operating the store result (a) from round-trip inefficiency, which in the formulation of the present paper would be captured by the cost functions $C_{t}$ as defined by (3) with $C_{t}$ the same for all $t$, and (b) from buffering events, i.e. from failures to meet demand through insufficient energy available to be supplied from the store when it is needed, and from energy losses through store overflows. In the formulation of the present paper these costs would be captured by the functions $A_{t}$. In contrast to the present paper decisions affecting the level of the store (the amount of conventional generation to schedule for a particular time) are made $n$ time steps - rather than a single time step - in advance. The shocks to the system result from the differences between the available wind power as forecasted $n$ steps ahead of real time (when conventional generation is scheduled) and the wind power actually obtained. Although the model of the above papers is therefore not exactly the same as that of the present paper, the underlying arguments leading to Propositions 1-3 continue to apply, at least to a good approximation. In particular sample path arguments suggest that the reduction of roundtrip efficiency slows the rate at which the store-level trajectories - started from different initial levels but with the same stochastic description of future shock processes - converge over subsequent time.

Bejan et al [24] consider only the case where the round-trip efficiency is 1 . They study the efficiency of policies - analogous to those suggested by Proposition 1-whereby, for each $t$, the generation scheduled for time $t$ at the earlier time $t-n$ is such as would, given perfect forecasting, achieve a given target level $\hat{s}_{t}$ of the store at time $t$; this target level is independent of the level $s_{t-n}$ of the store at the time $t-n$ and of earlier scheduling decisions. However, Bejan et al [24] further take $\hat{s}_{t}$ to be independent of $t$, something which may not be optimal given the likely nonstationarity of the process of forecast errors.

Gast et al [25] subsequently study the same time series of available wind power, but allowed for round-trip efficiencies which are less than 1. They find (as might be expected here) that simple "target" policies such as that described above do not work well under these circumstances, and compare the behaviour of a variety of time-homogeneous policies.

\section{Determination of the functions $A_{t}$}

We described in Section 2 how, given a knowledge of the functions $A_{t}$, the optimal control of the store could be determined. In Sections 5-7 we develop such an approach, which is based 
on strong Lagrangian theory and which is very much more efficient, in senses explained there, than the application of standard dynamic programming or nonlinear optimisation techniques. In this section we consider conditions under which the functions $A_{t}$ may be thus known, either exactly or to good approximations.

Suppose that, as in Section 2, at the end of the time period $t-1$ the level of the store is $s_{t-1}$ and that, given $s_{t-1}$, any shocks prior to that time have no further effect on the optimal management of the store. Suppose further that an increase of $x_{t}$ (positive or negative) is planned for the time period $t$ (at a cost of $C_{t}\left(x_{t}\right)$ ). Recall that $A_{t}\left(s_{t-1}+x_{t}\right.$ ) is then defined to be the expected additional cost to the store of dealing optimally with any shock which may occur during the time period $t$, and may be conveniently characterised in terms of the coupling defined in that Section 2. Now define also $\bar{A}_{t}\left(s_{t-1}+x_{t}\right)$ to be the expected additional cost to the store of dealing with any shock which may occur during the time period $t$ and immediately returning the level of the store to its planned level $s_{t-1}+x_{t}$ at the end of the time period $t$. As in the case of the function $A_{t}$, we assume that each function $\bar{A}_{t}$ depends on $s_{t-1}$ and $x_{t}$ through their sum $s_{t-1}+x_{t}$ - the extent to which this approximation is reasonable being as discussed for the functions $A_{t}$. Given the costs of dealing with any shocks, and the known costs of making any immediate subsequent adjustments to the level of the store, the functions $\bar{A}_{t}$ are readily determinable, and in particular do not depend on how the store is controlled outside the time period $t$.

Note that, in the case of linear cost functions (i.e. $C_{t}(x)=c_{t} x$ for all $t$ ) and when shocks do not have effects which persist beyond the end of the time period in which they occur, the argument of Proposition 1 implies immediately that $A_{t}=\bar{A}_{t}$ for all $t$ : the linearity of $C_{t}$ implies that, at the end of the time period $t-1$ and when the level of the store is then $s_{t-1}$, if $s_{t-1}+x_{t}$ is the optimal planned level of the store for the end of the time period $t$, then it remains the optimal level of the store for the end of that time period following any shock which occurs during it.

More generally the functions $\bar{A}_{t}$ provide reasonable approximations to the functions $A_{t}$ to the extent to which it is reasonable, following any shock with which the store is required to deal, to return immediately the level of the store to that which would have obtained in the absence of the shock. In particular, when shocks are relatively rare but are potentially expensive (as might be the case when the store is required to pay the costs of failing to have sufficient energy to deal with an emergency), then the major contribution to both the functions $A_{t}$ and $\bar{A}_{t}$ will be this cost, regardless of precisely how the level of the store is adjusted in the immediate aftermath of the shock.

If necessary, better approximations to the functions $A_{t}$ may be obtained by allowing longer periods of time in which to optimally couple the trajectory of the store level, following a shock, to that which would have obtained in its absence. In applications one would wish to experiment a little here.

In applications there is also a need, when the costs of a shock arise from a failure to have insufficient energy in the store to deal with it, to identify what these costs are. There are various possible candidates. Two simple such - natural in the context of risk metrics for power systems, where they correspond respectively to loss of load and energy unserved (see, for example, [31]) - are:

(i) for each $t>0$, the cost of a shock occurring during the time period $t$ is simply some constant $a_{t}>0$ if there is insufficient energy within the store to meet it, and is otherwise 0 .

(ii) for each $t>0$, the cost of a shock occurring during the time period $t$ is proportional 
to the shortfall in the energy necessary to meet that shock.

Given the planned level $s_{t-1}+x_{t}$ of the store to be achieved during any time period $t$, the total additional cost of dealing with any shock occurring during that time period (as defined for example in terms of the coupling introduced in Section 2) is a random variable which is a function of the size of the shock. The distribution of this random variable, and so also its expectation $A_{t}\left(s_{t-1}+x_{t}\right)$ may need to be determined by observation.

Note finally that the effects of shocks may persist over several time periods (as, for example, when the store is required to provide ongoing support for the sudden loss of major piece of equipment such as a generator), so that each of the functions $A_{t}$-which will in general be decreasing - need not be flat for values of its argument in excess of the output rate constraint $P_{O t}$. In particular a reasonable way of dealing with a shock whose effects do persist over several time periods may simply be to reserve notionally sufficient energy in the store to deal with it; then, following such a shock, the level of the store will temporally become the excess over that reserve and the capacity of the store will correspondingly be temporally reduced. This causes no problems for the present theory, and is a reason for allowing a possible time dependence (which may be dynamic) for the capacity of the store. We consider some plausible functional forms of the functions $A_{t}$ in Section 8 .

\section{The optimal control problem}

We now assume that the functions $A_{t}$ defined in Section 2 are known, at least to a sufficiently good approximation - see the discussion of the previous section.

Define (the random variable) $\hat{s}=\left(\hat{s}_{0}, \ldots, \hat{s}_{T}\right)$ (with $\left.\hat{s}_{0}=s_{0}^{*}\right)$ to be the levels of the store at the end of the successive time periods $t=0, \ldots, T$ under the (stochastic) optimal control as defined in Section 2. Recall also from Section 2 that, for each $t$ and each level $s_{t-1}$ of the store at the end of the time period $t-1$, the quantity $\hat{x}_{t}\left(s_{t-1}\right)$ is the value of $x_{t} \in X_{t}$ which achieves the minimisation in the recursion (1).

For any vector $s=\left(s_{0}, \ldots, s_{T}\right)$ and for each $t=1, \ldots, T$, define

$$
x_{t}(s)=s_{t}-s_{t-1} \text {. }
$$

Define also the following (deterministic) optimisation problem:

P: choose $s=\left(s_{0}, \ldots, s_{T}\right)$ with $s_{0}=s_{0}^{*}$ so as to minimise

$$
\sum_{t=1}^{T}\left[C_{t}\left(x_{t}(s)\right)+A_{t}\left(s_{t}\right)\right]
$$

subject to the capacity constraints

$$
0 \leq s_{t} \leq E_{t}, \quad 1 \leq t \leq T
$$

and the rate constraints

$$
x_{t}(s) \in X_{t}, \quad 1 \leq t \leq T .
$$

Let $s^{*}=\left(s_{0}^{*}, \ldots, s_{T}^{*}\right)$ denote the solution to the above problem $\mathbf{P}$. It follows from direct iteration of the recursion (1), using also (2), that $x_{1}\left(s^{*}\right)$ achieves the minimisation in (1) for $t=1$ and when $s_{0}=s_{0}^{*}$, i.e. that $\hat{x}_{1}\left(s_{0}^{*}\right)=\hat{x}_{1}\left(\hat{s}_{0}\right)=x_{1}\left(s^{*}\right)$. Thus, from (11), provided no shock occurs during the time period 1 so that $\hat{s}_{1}=\hat{s}_{0}+\hat{x}_{1}\left(\hat{s}_{0}\right)$, we have also that $\hat{s}_{1}=s_{1}^{*}$. More generally, let the random variable $T^{\prime}$ index the first time period during which a shock does occur. Then repeated application of the above argument gives immediately the following result. 
Proposition 4. For all $t<T^{\prime}$, we have $\hat{s}_{t}=s_{t}^{*}$.

The solution to the problem $\mathbf{P}$ therefore defines the optimal control of the store up to the end of the time period $T^{\prime}$ defined above. At that time, and the end of each subsequent time period during which there occurs a shock, it is of course necessary to reformulate the problem $\mathbf{P}$, starting at the end of the time period $T^{\prime}$ (or as soon any shock occurring during that time period has been fully dealt with), instead of at time 0 , and replacing the initial level $s_{0}^{*}=\hat{s}_{0}$ by the perturbed level $\hat{s}_{T^{\prime}}$ of the store at that time. Thus the stochastic optimal control problem may be solved dynamically by the solution of the problem $\mathbf{P}$ at time 0 , and the further solution of (a reformulated version) of this problem at the end of each subsequent time period in which a shock occurs. The solution of the problem, which we now consider, is very much simpler than that of the corresponding stochastic dynamic programming approach.

\section{Lagrangian theory and characterisation of solution}

We showed in the previous section that, to the extent that the functions $A_{t}$ are known, an optimal control for the store may be developed via the solution of the optimisation problem $\mathbf{P}$ defined there. In Section 4 we discussed how to make what are in many cases good and readily determinable approximations for the functions $A_{t}$.

We again assume convexity of the functions $A_{t}$ (see Section 3), in addition to that of the functions $C_{t}$. We develop the strong Lagrangian theory $[32,33]$ associated with the problem $\mathbf{P}$. This leads to both an efficient algorithm for its solution, and to the identification of the Lagrange multipliers necessary for the proper dimensioning of the store. In particular Theorem 5 establishes the existence of a pair of vectors $\left(s^{*}, \lambda^{*}\right)$ such that $s^{*}$ solves the problem $\mathbf{P}$ and $\lambda^{*}$ is a function of the associated Lagrange multipliers corresponding to the capacity constraints (see below); the theorem further gives conditions necessarily satisfied by the pair $\left(s^{*}, \lambda^{*}\right)$.

We now introduce the more general problem $\mathbf{P}(a, b)$ in which $s_{0}$ is kept fixed at the value $s_{0}^{*}$ of interest above, but in which $s_{1}, \ldots, s_{T}$ are allowed to vary between quite general upper and lower bounds:

$\mathbf{P}(a, b)$ : choose $s=\left(s_{0}, \ldots, s_{T}\right)$, with $s_{0}=s_{0}^{*}$ so as to minimise

$$
\sum_{t=1}^{T}\left[C_{t}\left(x_{t}(s)\right)+A_{t}\left(s_{t}\right)\right]
$$

subject to the capacity constraints

$$
a_{t} \leq s_{t} \leq b_{t}, \quad 1 \leq t \leq T
$$

and the rate constraints

$$
x_{t}(s) \in X_{t}, \quad 1 \leq t \leq T .
$$

Here $a=\left(a_{1}, \ldots, a_{T}\right)$ and $b=\left(b_{1}, \ldots, b_{T}\right)$ are such that $a_{t} \leq b_{t}$ for all $t$. Let also $a^{*}$ and $b^{*}$ be the values of $a$ and $b$ corresponding to our particular problem $\mathbf{P}$ of interest, i.e.

$$
a_{t}^{*}=0, \quad b_{t}^{*}=E_{t}, \quad 1 \leq t \leq T .
$$

Note that the convexity of the functions $C_{t}$ and $A_{t}$ guarantees their continuity, and, since for each $a, b$ as above the space of allowed values of $s$ is compact, a solution $s^{*}(a, b)$ to 
the problem $\mathbf{P}(a, b)$ always exists. Let $V(a, b)$ be the corresponding minimised value of the objective function, i.e.

$$
V(a, b)=\sum_{t=1}^{T}\left[C_{t}\left(x_{t}\left(s^{*}(a, b)\right)\right)+A_{t}\left(s^{*}(a, b)\right)\right] .
$$

Observe also that the function $V(a, b)$ is itself convex in $a$ and $b$. To see this, consider any convex combination $(\bar{a}, \bar{b})=\left(\kappa a_{1}+(1-\kappa) a_{2}, \kappa b_{1}+(1-\kappa) b_{2}\right)$ of any two values $\left(a_{1}, b_{1}\right)$, $\left(a_{2}, b_{2}\right)$ of the pair $(a, b)$, where $0 \leq \kappa \leq 1$; since the constraints (16) and (17) are linear, it follows that the vector $\bar{s}=\kappa s^{*}\left(a_{1}, b_{1}\right)+(1-\kappa) s^{*}\left(a_{2}, b_{2}\right)$ is feasible for the problem $\mathbf{P}(\bar{a}, \bar{b})$; hence, from the convexity of the functions $C_{t}$ and $A_{t}$,

$$
\begin{aligned}
V(\bar{a}, \bar{b}) & \leq \sum_{t=1}^{T}\left[C_{t}\left(x_{t}(\bar{s})\right)+A_{t}\left(\bar{s}_{t}\right)\right] \\
& =\sum_{t=1}^{T}\left[C_{t}\left(\kappa x_{t}\left(s^{*}\left(a_{1}, b_{1}\right)\right)+(1-\kappa) x_{t}\left(s^{*}\left(a_{2}, b_{2}\right)\right)\right)+A_{t}\left(\kappa s_{t}^{*}\left(a_{1}, b_{1}\right)+(1-\kappa) s_{t}^{*}\left(a_{2}, b_{2}\right)\right)\right] \\
& \leq \kappa \sum_{t=1}^{T}\left[C_{t}\left(x_{t}\left(s^{*}\left(a_{1}, b_{1}\right)\right)\right)+A_{t}\left(s_{t}^{*}\left(a_{1}, b_{1}\right)\right)\right]+(1-\kappa) \sum_{t=1}^{T}\left[C_{t}\left(x_{t}\left(s^{*}\left(a_{2}, b_{2}\right)\right)\right)+A_{t}\left(s_{t}^{*}\left(a_{2}, b_{2}\right)\right)\right] \\
& =\kappa V\left(a_{1}, b_{1}\right)+(1-\kappa) V\left(a_{2}, b_{2}\right) .
\end{aligned}
$$

We now have the following result, which encapsulates the relevant strong Lagrangian theory.

Theorem 5. Let $s^{*}$ denote the solution to the problem $\mathbf{P}$. Then there exists a vector $\lambda^{*}=\left(\lambda_{1}^{*}, \ldots, \lambda_{T}^{*}\right)$ such that

(i) for all vectors $s$ such that $s_{0}=s_{0}^{*}$ and $x_{t}(s) \in X_{t}$ for all $t$ ( $s$ is not otherwise constrained),

$$
\sum_{t=1}^{T}\left[C_{t}\left(x_{t}(s)\right)+A_{t}\left(s_{t}\right)-\lambda_{t}^{*} s_{t}\right] \geq \sum_{t=1}^{T}\left[C_{t}\left(x_{t}\left(s^{*}\right)\right)+A_{t}\left(s_{t}^{*}\right)-\lambda_{t}^{*} s_{t}^{*}\right] .
$$

(ii) the pair $\left(s^{*}, \lambda^{*}\right)$ satisfies the complementary slackness conditions, for $1 \leq t \leq T$,

$$
\begin{cases}\lambda_{t}^{*}=0 & \text { if } 0<s_{t}^{*}<E_{t} \\ \lambda_{t}^{*} \geq 0 & \text { if } s_{t}^{*}=0 \\ \lambda_{t}^{*} \leq 0 & \text { if } s_{t}^{*}=E_{t} .\end{cases}
$$

Conversely, suppose that there exists a pair of vectors $\left(s^{*}, \lambda^{*}\right)$, with $s_{0}=s_{0}^{*}$, satisfying the conditions (i) and (ii) and such that $s^{*}$ is additionally feasible for the problem $\mathbf{P}$. Then $s^{*}$ solves the problem $\mathbf{P}$.

Proof. Consider the general problem $\mathbf{P}(a, b)$ defined above. Introduce slack (or surplus) variables $z=\left(z_{1}, \ldots, z_{t}\right)$ and $w=\left(w_{1}, \ldots, w_{t}\right)$ and rewrite $\mathbf{P}(a, b)$ as:

$\mathbf{P}(a, b)$ : minimise $\sum_{t=1}^{T}\left[C_{t}\left(x_{t}(s)\right)+A_{t}\left(s_{t}\right)\right]$ over all $s=\left(s_{0}, \ldots, s_{T}\right)$ with $s_{0}=s_{0}^{*}$, over all $z \geq 0$, over all $w \geq 0$, and subject to the further constraints

$$
\begin{aligned}
& s_{t}-z_{t}=a_{t}, \quad 1 \leq t \leq T, \\
& s_{t}+w_{t}=b_{t}, \quad 1 \leq t \leq T,
\end{aligned}
$$

and also $x_{t}(s) \in X_{t}$ for $1 \leq t \leq T$. 
Since the function $V(a, b)$ is also convex in $a$ and $b$, it follows from the supporting hyperplane theorem (see [32] or [33]), that there exist Lagrange multipliers $\alpha^{*}=\left(\alpha_{1}^{*}, \ldots, \alpha_{T}^{*}\right)$ and $\beta^{*}=\left(\beta_{1}^{*}, \ldots, \beta_{T}^{*}\right)$ such that

$$
V(a, b) \geq V\left(a^{*}, b^{*}\right)+\sum_{t=1}^{T} \alpha_{t}^{*}\left(a_{t}-a_{t}^{*}\right)+\sum_{t=1}^{T} \beta_{t}^{*}\left(b_{t}-b_{t}^{*}\right) \quad \text { for all } a, b
$$

Thus also, for all $s$ with $s_{0}=s_{0}^{*}$ and such that $x_{t}(s) \in X_{t}$ for $1 \leq t \leq T$, for all $z \geq 0$, and for all $w \geq 0$, by defining $a$ and $b$ via (21) and (22), we have

$$
\begin{aligned}
\sum_{t=1}^{T}\left[C_{t}\left(x_{t}(s)\right)+A_{t}\left(s_{t}\right)-\alpha_{t}^{*}\left(s_{t}-z_{t}\right)-\right. & \left.\beta_{t}^{*}\left(s_{t}+w_{t}\right)\right] \\
& \geq \sum_{t=1}^{T}\left[C_{t}\left(x_{t}\left(s^{*}\right)\right)+A_{t}\left(s_{t}^{*}\right)-\alpha_{t}^{*} a_{t}^{*}-\beta_{t}^{*} b_{t}^{*}\right] .
\end{aligned}
$$

Since the components of $z$ and $w$ may take arbitrary positive values, we obtain at once the following complementary slackness conditions for the vectors of Lagrange multipliers $\alpha^{*}$ and $\beta^{*}$ :

$$
\begin{array}{lll}
\alpha_{t}^{*} \geq 0, & \alpha_{t}^{*}=0 \text { whenever } s_{t}^{*}>a_{t}^{*}, & 1 \leq t \leq T, \\
\beta_{t}^{*} \leq 0, & \beta_{t}^{*}=0 \text { whenever } s_{t}^{*}<b_{t}^{*}, & 1 \leq t \leq T .
\end{array}
$$

Thus, from (24)-(26), by taking $z_{t}=w_{t}=0$ for all $t$ on the left side of (24), it follows that, for all $s$ with $s_{0}=s_{0}^{*}$ and $x_{t}(s) \in X_{t}$ for $1 \leq t \leq T$,

$$
\sum_{t=1}^{T}\left[C_{t}\left(x_{t}(s)\right)+A_{t}\left(s_{t}\right)-\left(\alpha_{t}^{*}+\beta_{t}^{*}\right) s_{t}\right] \geq \sum_{t=1}^{T}\left[C_{t}\left(x_{t}\left(s^{*}\right)\right)+A_{t}\left(s_{t}^{*}\right)-\left(\alpha_{t}^{*}+\beta_{t}^{*}\right) s_{t}^{*}\right] .
$$

The condition (i) of the theorem now follows on defining

$$
\lambda_{t}^{*}=\alpha_{t}^{*}+\beta_{t}^{*}, \quad 1 \leq t \leq T .
$$

while the condition (ii) follows from (28) on using also the complementary slackness conditions (25) and (26).

To prove the converse result, suppose that a pair $\left(s^{*}, \lambda^{*}\right)$ (with $\left.s_{0}=s_{0}^{*}\right)$ satisfies the conditions (i) and (ii) and that $s^{*}$ is feasible for the problem $\mathbf{P}$. From the condition (ii), we may define (unique) vectors $\alpha^{*}=\left(\alpha_{1}^{*}, \ldots, \alpha_{T}^{*}\right)$ and $\beta^{*}=\left(\beta_{1}^{*}, \ldots, \beta_{T}^{*}\right)$ such that the conditions (25), (26) and (28) hold. The condition (i) of the theorem now translates to the requirement that, for all vectors $s$ such that $s_{0}=s_{0}^{*}$ and $x_{t}(s) \in X_{t}$ for all $t$, the relation (27) holds. Finally, it follows from this and from the conditions (25) and (26) that, for any vector $s$ which is feasible for the problem $\mathbf{P}$ - and so in particular satisfies $0 \leq s_{t} \leq E_{t}$ for all $t$,

$$
\sum_{t=1}^{T}\left[C_{t}\left(x_{t}(s)\right)+A_{t}\left(s_{t}\right)\right] \geq \sum_{t=1}^{T}\left[C_{t}\left(x_{t}\left(s^{*}\right)\right)+A_{t}\left(s_{t}^{*}\right)\right],
$$

so that $s^{*}$ solves the problem $\mathbf{P}$ as required.

Remark 5. Note that the second part of Theorem 5, i.e. the converse result, does not require the convexity assumptions on the functions $C_{t}$ and $A_{t}$. 
The above Lagrangian theory - which we require for the determination of the optimal control as described in Section 7-further enables a determination of the sensitivity of the value of the store with respect to variation of its capacity constraints. For the given problem $\mathbf{P}$, the cost of optimally operating the store (the negative of its value) is given by $V\left(a^{*}, b^{*}\right)$, where we recall that $a^{*}$ and $b^{*}$ are as given by (18). For any $t$, the derivative of this optimised cost with respect to $E_{t}$, assuming this derivative to exist, is given by the Lagrange multiplier $\beta_{t}^{*}$ defined in the above proof (the differentiability assumption ensuring that $\beta_{t}$ is here uniquely defined). Note further that when $s_{t}^{*}<E_{t}$ then (from (26)) the Lagrange multiplier $\beta_{t}^{*}$ is equal to zero, and when $s_{t}^{*}=E_{t}$ then (from (25) and (28)) we have $\beta_{t}^{*}=\lambda_{t}^{*}$.

A further determination of the sensitivity of the value of the store with respect to variation of its rate constraint may be developed along the lines of Theorem 5 of Cruise et al [5], but we do not pursue this here.

\section{$7 \quad$ Determination of $\left(s^{*}, \lambda^{*}\right)$}

The structure of the objective function causes some difficulties for the solution of the problem $\mathbf{P}$. As previously observed, a dynamic programming approach might seem natural but, even for this deterministic problem, typically remains too computationally complexon account of both the likely time-heterogeneity of the functions $A_{t}$ and $C_{t}$, and of the need, even for small $t$, to consider the problem over the entire time interval $[0, T]$.

We continue to assume convexity of the functions $C_{t}$ and $A_{t}$. Under the further assumption of differentiability of the functions $A_{t}$, we give an efficient algorithm for the construction of a pair $\left(s^{*}, \lambda^{*}\right)$ satisfying the conditions of Theorem 5- so that, in particular, $s^{*}$ solves the problem $\mathbf{P}$. This algorithm is further sequential and local in time, in the sense that the determination of the solution to any given time $t^{\prime} \leq T$ typically requires only the consideration of the problem, i.e. a knowledge of the functions $C_{t}$ and $A_{t}$, for those times $t$ extending to some time horizon which is typically only a short distance beyond $t^{\prime}$. We have already shown in Section 5 that the ability to dynamically solve the deterministic problem $\mathbf{P}$, or updates of this problem, at the times of successive shocks enables an efficient (stochastically) optimal control of the store.

We give conditions necessarily satisfied by the pair $\left(s^{*}, \lambda^{*}\right)$. Under the further assumption of strict convexity of the functions $C_{t}$, we show how these conditions may be used to determine $\left(s^{*}, \lambda^{*}\right)$ uniquely. We then indicate how the strict/ convexity assumption may be relaxed.

Proposition 6. Suppose that the functions $A_{t}$ are differentiable, and that the pair $\left(s^{*}, \lambda^{*}\right)$ is such that $s^{*}$ is feasible for the problem $\mathbf{P}$, while $\left(s^{*}, \lambda^{*}\right)$ satisfies the condition (ii) of Theorem 5. For each $t$ define

$$
\nu_{t}^{*}=\sum_{u=t}^{T}\left[\lambda_{u}^{*}-A_{u}^{\prime}\left(s_{u}^{*}\right)\right] .
$$

Then the condition that $\left(s^{*}, \lambda^{*}\right)$ satisfies the condition (i) of Theorem 5 is equivalent to the condition that

$$
x_{t}\left(s^{*}\right) \text { minimises } C_{t}(x)-\nu_{t}^{*} x \text { in } x \in X_{t}, \quad 1 \leq t \leq T \text {. }
$$


Proof. Assume that the pair $\left(s^{*}, \lambda^{*}\right)$ is as given. Suppose first that additionally $\left(s^{*}, \lambda^{*}\right)$ satisfies the condition (i) of Theorem 5 . The condition (31) is then straightforward when the functions $C_{t}$ are additionally differentiable: for each $t$ the partial derivative of the left side of (19) with respect to $x_{t}(s)$ (with $x_{u}(s)$ being kept constant for $u \neq t$ ) is necessarily zero at $s=s^{*}$, so that (31) follows from the assumed convexity of the functions $C_{t}$. For the general case, note that it follows from the condition (i) of Theorem 5 (by considering $s$ such that $s_{0}=s_{0}^{*}, x_{t}(s)=x_{t}\left(s^{*}\right)+h, x_{u}(s)=x_{u}\left(s^{*}\right)$ for $\left.u \neq t\right)$, that, for all $t$ and for all real $h$,

$$
C_{t}\left(x_{t}\left(s^{*}\right)+h\right)+\sum_{u=t}^{T}\left[A_{u}\left(s_{u}^{*}+h\right)-\lambda_{u}^{*} h\right]
$$

is minimised at $h=0$, and so, for all (small) $h$,

$$
C_{t}\left(x_{t}\left(s^{*}\right)+h\right)-\nu_{t} h \geq C_{t}\left(x_{t}\left(s^{*}\right)\right)+o(h), \quad \text { as } h \rightarrow 0 .
$$

Thus (31) again follows from the assumed convexity of the functions $C_{t}$.

To prove the converse result, suppose now that $\left(s^{*}, \lambda^{*}\right)$ satisfies the condition (31). This condition, together with the convexity and differentiability of the functions $A_{t}$, then implies that, for all $t$, the expression (32) is minimised at $h=0$. It is now straightforward that the hyperplane in $\mathbb{R}^{T}$ whose vector of slopes is $\lambda^{*}$ supports the function $\sum_{t=1}^{T}\left[C_{t}\left(x_{t}(s)\right)+A_{t}\left(s_{t}\right)\right]$ at the point $\left(s^{*}, \sum_{t=1}^{T}\left[C_{t}\left(x_{t}\left(s^{*}\right)\right)+A_{t}\left(s_{t}^{*}\right)\right]\right)$, so that finally the condition (i) of Theorem 5 holds as required.

It now follows from Theorem 5 and Proposition 6 that if the pair $\left(s^{*}, \lambda^{*}\right)$ is such that $s^{*}$ is feasible for the problem $\mathbf{P}$, and that $\left(s^{*}, \lambda^{*}\right)$ satisfies the both condition (31) and the condition (ii) of Theorem 5 , then $s^{*}$ further solves the problem $\mathbf{P}$.

We now show how to construct such a pair $\left(s^{*}, \lambda^{*}\right)$. We assume, for the moment, strict convexity of the functions $C_{t}$; we subsequently indicate how to relax this assumption. It follows from the assumed strict convexity that, for each $t$ and for each $\nu_{t}$, there is a unique $x \in X_{t}$, which we denote by $x_{t}^{*}\left(\nu_{t}\right)$, which minimises $C_{t}(x)-\nu_{t} x$ in $X_{t}$. Further $x_{t}^{*}\left(\nu_{t}\right)$ is continuous and increasing in $\nu_{t}$-strictly so for $\nu_{t}$ such that $x_{t}^{*}\left(\nu_{t}\right)$ lies in the interior of $X_{t}$. In particular, from (11), the condition (31) may now be rewritten as

$$
s_{t}^{*}=s_{t-1}^{*}+x_{t}^{*}\left(\nu_{t}^{*}\right), \quad 1 \leq t \leq T .
$$

It further follows from (30) that

$$
\nu_{t+1}^{*}=\nu_{t}^{*}+A_{t}^{\prime}\left(s_{t}^{*}\right)-\lambda_{t}^{*} . \quad 1 \leq t \leq T-1 .
$$

Thus, were the vector $\lambda^{*}$ known, together with the value of the constant $\nu_{1}^{*}$, the pair $\left(s^{*}, \nu^{*}\right)$ could be constructed sequentially via (34) and (35). We observe that, while $\lambda^{*}$ is not known, it does satisfy the conditions (20) and in particular the requirement that $\lambda_{t}^{*}=0$ for all $t$ such that $0<s_{t}^{*}<E_{t}$. We now follow a procedure which is a generalisation of one described by Cruise et al [5], and which involves an essentially onedimensional search so as to identify the constant $\nu_{1}^{*}$. This search, which may be thought of as being carried out at time zero and which is not computationally intensive (see the further remarks at the end of this section), then needs to be repeated at each of a number of subsequent times as described below. We show how to define inductively a sequence of times $0=T_{0}<T_{1}<\cdots<T_{k}=T$ such that $s^{*}\left(T_{i}\right)=0$ or $s^{*}\left(T_{i}\right)=E_{T_{i}}$ for $1 \leq i \leq k$ and such that $\lambda_{t}^{*}=0$ for all values of $t$ not in the above sequence. 
The time $T_{1}$ is chosen as follows. Consider trial values $\nu_{1}$ of $\nu_{1}^{*}$. For each such $\nu_{1}$, define a pair of vectors $\nu=\left(\nu_{1}, \ldots, \nu_{T}\right)$ and $s=\left(s_{1}, \ldots, s_{T}\right)$ by

$$
\begin{array}{cc}
s_{t} & =s_{t-1}+x_{t}^{*}\left(\nu_{t}\right), \quad 1 \leq t \leq T, \\
\nu_{t+1} & =\nu_{t}+A_{t}^{\prime}\left(s_{t}\right), \quad 1 \leq t \leq T-1 .
\end{array}
$$

Define $M$ and $M^{\prime}$ to be the sets of values of $\nu_{1}$ for which the vector $s$ defined via (36) and (37) violates one of the capacity constraints (13) and first does so respectively below or above - in either case at a time which we denote by $\bar{T}_{1}\left(\nu_{1}\right)$. Since, for each $t, x_{t}^{*}\left(\nu_{t}\right)$ is increasing in $\nu_{t}$ and $A_{t}^{\prime}\left(s_{t}\right)$ is increasing in $s_{t}$ (by the convexity of $A_{t}$ ), it follows that if $\nu_{1} \in M$ then $\nu_{1}^{\prime} \in M$ for all $\nu_{1}^{\prime}<\nu_{1}$ and that if $\nu_{1} \in M^{\prime}$ then $\nu_{1}^{\prime} \in M^{\prime}$ for all $\nu_{1}^{\prime}>\nu_{1}$; further the sets $M$ and $M^{\prime}$ are disjoint, and (since the solution set for the problem $\mathbf{P}$ is nonempty) neither the set $M$ nor the set $M^{\prime}$ can be the entire real line. Let $\bar{\nu}_{1}=\sup M$. (In the extreme case where $M$ is empty we may set $\bar{\nu}_{1}=-\infty$ ). We now consider the behaviour of the corresponding vector $s$ defined via (36) and (37) where we take $\nu_{1}=\bar{\nu}_{1}$; for this vector $s$ there are three possibilities:

(a) the quantity $\bar{\nu}_{1}$ belongs neither to the set $M$ nor to the set $M^{\prime}$, i.e. the vector $s$ generated as above is feasible for the problem $\mathbf{P}$; in this case we take $T_{1}=T$ and $s^{*}=s$ with $\nu_{1}^{*}=\bar{\nu}_{1}$ and $\lambda_{t}^{*}=0$ for $1 \leq t \leq T-1$ (so that the remaining values of $\nu^{*}$ are given by (35));

(b) the quantity $\bar{\nu}_{1}$ belongs to the set $M$; in this case there exists at least one $t<\bar{T}_{1}\left(\bar{\nu}_{1}\right)$ such that $s_{t}=E_{t}$ (were this not so then, by the continuity of each $x_{t}^{*}\left(\nu_{t}\right)$ in $\nu_{t}$, the value of $\nu_{1}$ could be increased above $\bar{\nu}_{1}$ while remaining within the set $M$ ); define $T_{1}$ to be any such $t$, say the largest, and take $s_{t}^{*}=s_{t}$ for $1 \leq t \leq T_{1}$ with $\nu_{1}^{*}=\bar{\nu}_{1}$ and $\lambda_{t}^{*}=0$ for $1 \leq t \leq T_{1}-1$;

(c) the quantity $\bar{\nu}_{1}$ belongs to the set $M^{\prime}$; in this case, similarly to the case (b), there exists at least one $t<\bar{T}_{1}\left(\bar{\nu}_{1}\right)$ such that $s_{t}=0$; define $T_{1}$ to be any such $t$, again say the largest, and again take $s_{t}^{*}=s_{t}$ for $1 \leq t \leq T_{1}$ with $\nu_{1}^{*}=\bar{\nu}_{1}$ and $\lambda_{t}^{*}=0$ for $1 \leq t \leq T_{1}-1$.

In each of the cases (b) and (c) above, we now repeat the above procedure, starting at the time $T_{1}$ instead of the time 0 , and considering trial values of $\nu_{T_{1}+1}^{*}$, thereby identifying $\nu_{T_{1}+1}^{*}$, the time $T_{2}$ and the values of $s_{t}^{*}$ for $T_{1}+1 \leq t \leq T_{2}$, and taking $\lambda_{t}^{*}=0$ for $T_{1}+1 \leq t \leq T_{2}-1$. The quantity $\lambda_{T_{1}}^{*}$ is now defined via (35). Further consideration of the sets $M$ and $M^{\prime}$ defined above in relation to the identification of $\nu_{1}^{*}=\bar{\nu}_{1}$ shows easily that in the case $\bar{\nu}_{1} \in M$ - so that $s_{T_{1}}^{*}=E_{T_{1}}$ - the quantity $\nu_{T_{1}+1}^{*}=\bar{\nu}_{T_{1}+1}$ is necessarily such that $\lambda_{T_{1}}^{*} \geq 0$ (since in this case, by the above construction, the quantity $\nu_{T_{1}+1}^{*}$ has a value which is necessarily at least as great as would have been the case had $\lambda_{T_{1}}^{*}$ been equal to 0 ), whereas in the case $\bar{\nu}_{1} \in M^{\prime}$ - so that $s_{T_{1}}^{*}=0$ - the quantity $\nu_{T_{1}+1}^{*}=\bar{\nu}_{T_{1}+1}$ is necessarily such that $\lambda_{T_{1}}^{*} \leq 0$.

For $T_{2} \neq T$ we continue in this manner until the entire sequence $0=T_{0}<T_{1}<\cdots<$ $T_{k}=T$ is identified. We thus obtain vectors $s^{*}, \lambda^{*}$ and $\nu^{*}$ such that $s^{*}$ is feasible for the problem $\mathbf{P}$, while $\left(s^{*}, \lambda^{*}\right)$ satisfies the condition (31) and the condition (ii) of Theorem 5 and so solves the problem $\mathbf{P}$ as required.

In the case where, for at least some $t$, the cost function $C_{t}$ is convex, but not necessarily strictly so, some extra care is required. Here, for such $t$, the function $\nu \rightarrow x_{t}^{*}(\nu)$ is not in general uniquely defined; further, for any given choice, this function is not in general continuous. However, the above construction of $\left(s^{*}, \lambda^{*}\right)$ continues to hold provided that, where necessary, we choose the right value of $x_{t}^{*}(\nu)$. The latter may always be identified 
by considering, for example, a sequence of strictly convex functions $C_{t}^{(\epsilon)}$ converging to $C_{t}$ and identifying $x_{t}^{*}(\nu)$ as the limit of its corresponding values within this sequence.

Note that the above construction proceeds locally in time, in the sense that, at each successive time $T_{i}$, the determination of the subsequent time $T_{i+1}$ and of the values of $s_{t}^{*}$ and $\nu_{t}^{*}$ for $T_{i}+1 \leq t \leq T_{i+1}$ only requires consideration of the functions $C_{t}$ and $A_{t}$ up to some time $\bar{T}_{i+1}$ (necessarily beyond $T_{i+1}$ ) the identification of which does not depend on the functions $C_{t}$ and $A_{t}$ at any subsequent times. More precisely we have $\bar{T}_{1}=\bar{T}_{1}\left(\bar{\nu}_{1}\right)$, where $\bar{T}_{1}\left(\bar{\nu}_{1}\right)$ is as identified above, and the remaining $\bar{T}_{i}, 2 \leq i \leq k$, are similarly identified. In particular we have that, for each time $t$ and given $s_{t-1}^{*}$, the optimal choice of store level $s_{t}^{*}$ depends only on the functions $C_{t^{\prime}}$ and $A_{t^{\prime}}$ for $t \leq t^{\prime} \leq \bar{T}(t)$ where we define $\bar{T}(t)=\bar{T}_{i+1}$ for $i$ such that $T_{i}+1 \leq t \leq T_{i+1}$. The function $\bar{T}(t)$ is piecewise constant in $t$, and so the time horizon or look-ahead time $\bar{T}(t)-t$ required for the optimal decision at each time $t$ has the "sawtooth" shape which we illustrate in our examples of Section 8 .

Note further that a lengthening of the total time $T$ over which the optimization is to be performed does not in general change the values of the times $T_{i}$, but rather simply creates more of them. In particular the solution to the problem $\mathbf{P}$ involves computation which grows essentially linearly in $T$, and the algorithm is suitable for the management of a store with an infinite time horizon.

The typical length of the intervals between the successive times $T_{i}$ depends on the shape of the functions $C_{t}$ and $A_{t}$ and in particular on the rate at which they fluctuate in time. Thus, for example, the long-run management of a store for which the functions $C_{t}$ show strong daily fluctuations typically involves decision making on a running time horizon of the order of a day or so.

Finally note that, as already indicated, in the implementation of the above construction, some form of one-dimensional search is usually required to determine each of the successive $\bar{\nu}_{T_{i}+1}$ : each trial value of this quantity provides either an upper or lower bound to the true value, so that, for example, a simple binary search is sufficient. Given also the "locality" property referred to above, the numerical effort involved in the implementation of the above algorithm is usually very slight.

\section{Examples}

We give some examples, in which we solve (exactly) the optimal control problem $\mathbf{P}$ formally defined in Section 5. We investigate how the optimal solution depends on the cost functions $C_{t}$ defined there which reflecting buying and selling costs and hence the opportunity to make money from price arbitrage, and on the functions $A_{t}$ which reflect the costs of providing buffering services.

The cost functions $C_{t}$ are derived from half-hourly electricity prices in the Great Britain spot market over the entire year 2011, adjusted for a modest degree of market impact, as described in detail below. Thus we work in half-hour time units, with the time horizon $T$ corresponding to the number of half-hour periods in the entire year. These spot market prices show a strong daily cyclical behaviour (corresponding to daily demand variation), being low at night and high during the day. This price variation can be seen in Figure 1 which shows half-hourly GB spot prices (in pounds per megawatt-hour) throughout the month of March 2011. There is a similar patter of variation throughout the rest of the year. 


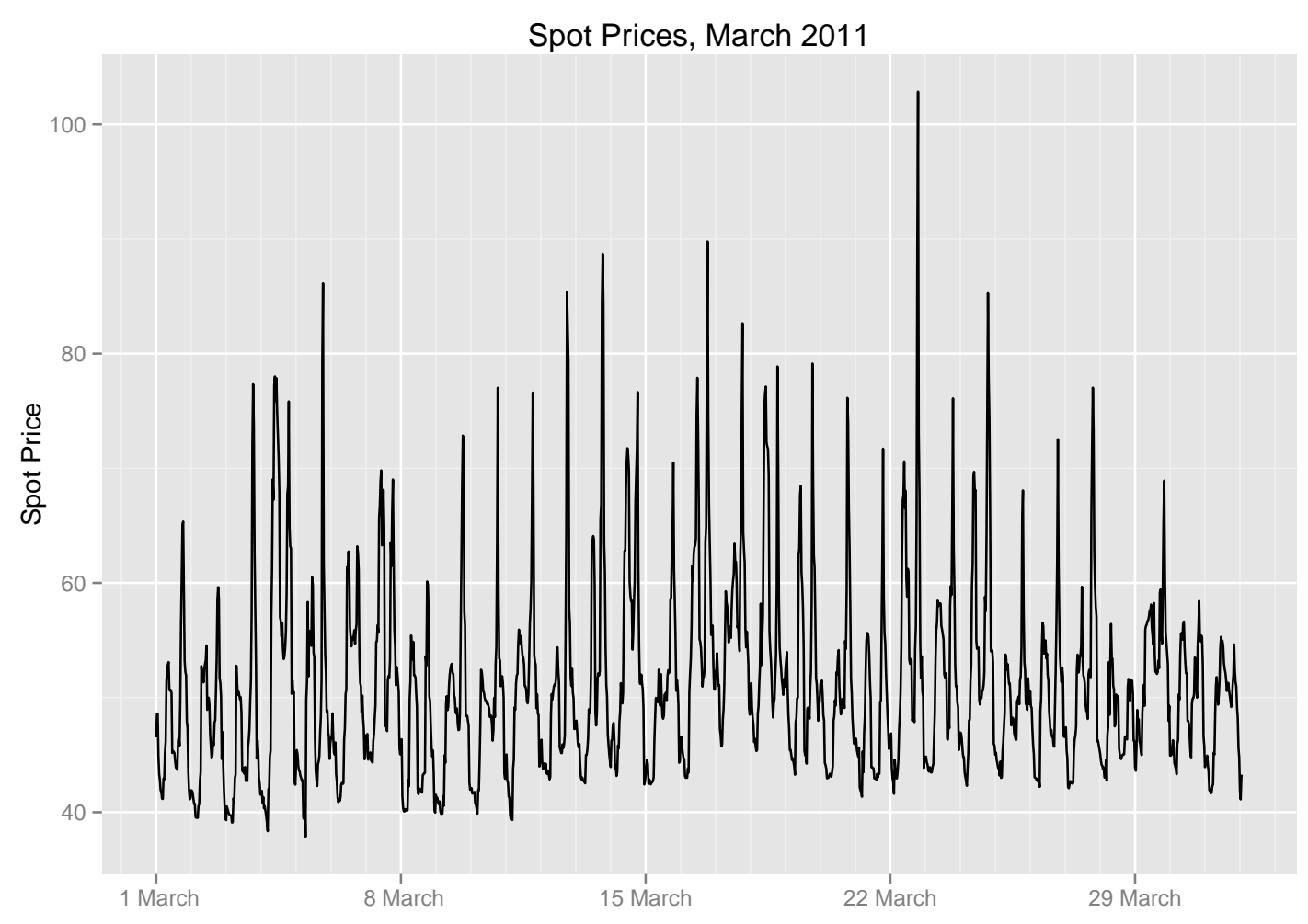

Figure 1: GB half-hourly spots prices (£/MWh) for March 2011.

Without loss of generality, we choose energy units such that the rate (power) constraints are given by $P_{I t}=P_{O t}=1$ unit of energy per half-hour period. For illustration, we take the capacity of the store to be given by $E=10$ units of energy; thus the store can completely fill or empty over a 5-hour period, which is the case, for example, for the large Dinorwig pumped storage facility in Snowdonia [34].

We choose cost functions $C_{t}$ of the form

$$
C_{t}(x)= \begin{cases}c_{t} x(1+\delta x), & \text { if } x \geq 0 \\ \eta c_{t} x(1+\delta x), & \text { if } x<0\end{cases}
$$

where the $c_{t}$ are proportional to the half-hourly electricity spot prices referred to above, where $\eta$ is an adjustment to selling prices representing in particular round-trip efficiency as described in Section 2, and where the factor $\delta>0$ is chosen so as to represent a degree of market impact (higher unit prices as the store buys more and lower unit prices as the store sells more). For our numerical examples we take $\eta=0.85$ which is a typical round-trip efficiency for a pumped-storage facility such as Dinorwig. We choose $\delta=0.05$; since the rate constraints for the store are $P_{I t}=P_{O t}=1$ this corresponds to a maximum market impact of $5 \%$. While this is modest, our results are qualitatively little affected as $\delta$ is varied over a wide range of values less than one, covering therefore the range of possible market impact likely to be seen for storage in practice.

Finally we need to choose the functions $A_{t}$ reflecting the costs of providing buffering services. Our aim here is to give an understanding of how the optimal control of the store varies according to the relative economic importance of cost arbitrage and buffering, i.e. according to the relative size of the functions $C_{t}$ and $A_{t}$. We choose functions $A_{t}$ which are constant over time $t$ and of the form $A_{t}(s)=a e^{-\kappa s}$ and $A_{t}(s)=b / s$ for a 
small selection of the parameters $a, \kappa$ and $b$. The extent to which a store might provide buffering services in applications is extremely varied, and so the likely balance between arbitrage and buffering cannot be specified in advance. Rather we choose just sufficient values of the above parameters to show the effect of varying this balance. For a possible justification of the chosen forms of the functions $A_{t}$ (including why it should not necessarily be truncated to 0 for values of $s$ greater than the rate constraint of 1 ), see Section 4 ; in particular the form $A_{t}(s)=a e^{-\kappa s}$ is plausible in the case of light-tailed shocks, while the form $A_{t}(s)=b / s$ shows the effect of a slow rate of decay in $s$.

In each of our examples, we determine the optimal control of the store over the entire year, with both the initial level $S_{0}^{*}$ and the final level $S_{T}^{*}$ given by $S_{0}^{*}=S_{T}^{*}=0$. In each of the corresponding figures, the upper panel shows the optimally controlled level of the store throughout the month of March. The lower panel shows, for each time $t$ in the same month, the time horizon (or look-ahead time) $\bar{T}(t)-t$, defined in Section 7, i.e. the length of time beyond the time $t$ for which knowledge of the cost functions is required in order to make the optimal decision at time $t$.

Figure 2 shows the optimal control of the store when the functions $A_{t}$ are given by $A_{t}(s)=$ $a e^{-\kappa s}$. The uppermost panels correspond to $a=0$, so that the store incurs no penalty for failing to provide buffering services and optimises its control solely on the basis of arbitrage between energy prices at different times. The daily cycle of prices is sufficiently pronounced that here the store fills and empties - or nearly so - on a daily basis, notwithstanding the facts that the round-trip efficiency of 0.85 is considerably less than 1 and that the minimum time for the store to fill or empty is 5 hours. It will be seen also that the time horizon, or look-ahead time, required for the determination of optimal decisions is in general of the order of one or two days.

The central panels of Figure 2 correspond to $\kappa=1$ and $a=1$. The choice of $a$ in particular is such that the store is just sufficiently incentivised by the need to reduce buffering costs that it rarely empties completely (though it does so very occasionally). Otherwise the behaviour of the store is very similar to that in the case $a=0$. Note also that in this case the time horizons or look-ahead times are in general somewhat longer; an intuitive explanation (backed by a careful examination of the figure) is that, starting from a time when the store is full, the determination of by how much the store should avoid emptying completely requires taking account of the cost functions for a longer period of future time than is the case where the store does empty completely.

Finally the bottom two panels of Figure 2 correspond to $\kappa=1$ and $a=10$. Here the costs of failing to provide buffering services are much higher, and so the optimised level of the store rarely falls below $25 \%$ of its capacity. Curiously the look-ahead times are in general less than in the case $a=1$-presumably since the store level is more often reaching the capacity constraint.

Variation of the exponential parameter $\kappa$ does not result in dramatically different behaviour, so we do not pursue this here.

Figure 3 shows the optimal control of the store when the functions $A_{t}$ are given, for each $t$, by $A_{t}(s)=b / s$. The upper panels correspond to $b=0$, so that we again have $A_{t}(s)=0$ for all $s$ and the control is as observed previously. The lower panels correspond to the case $b=1$, and, as might be expected, the behaviour here is somewhat intermediate between that for the two nonzero exponentially decaying exponential functions. 
$A(S)=0$

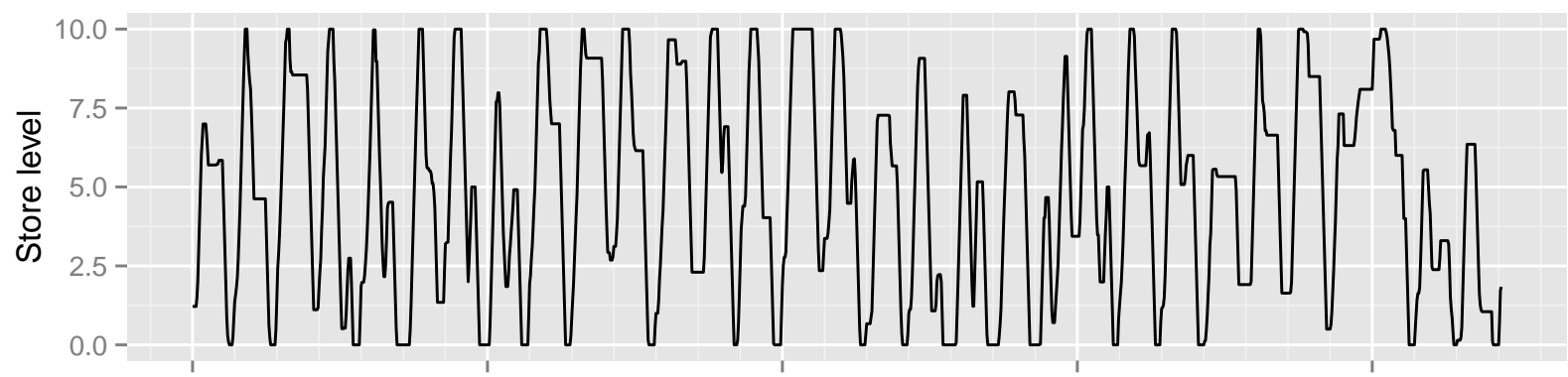

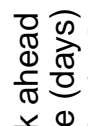

농

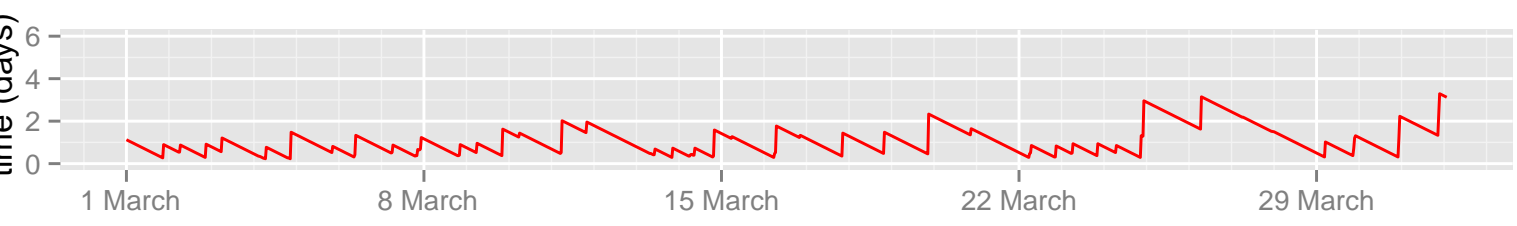

$A(S)=e^{-S}$

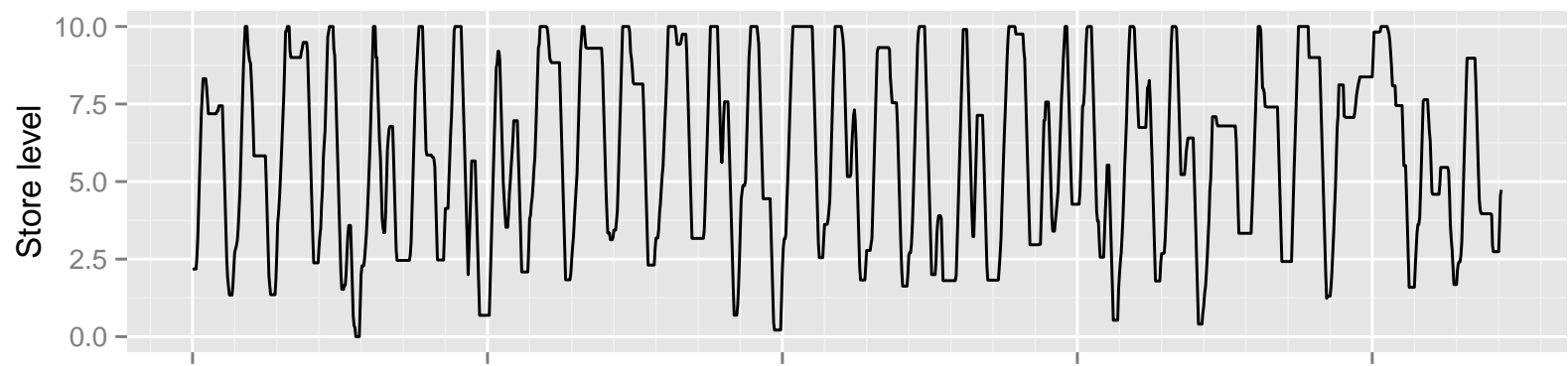

1.
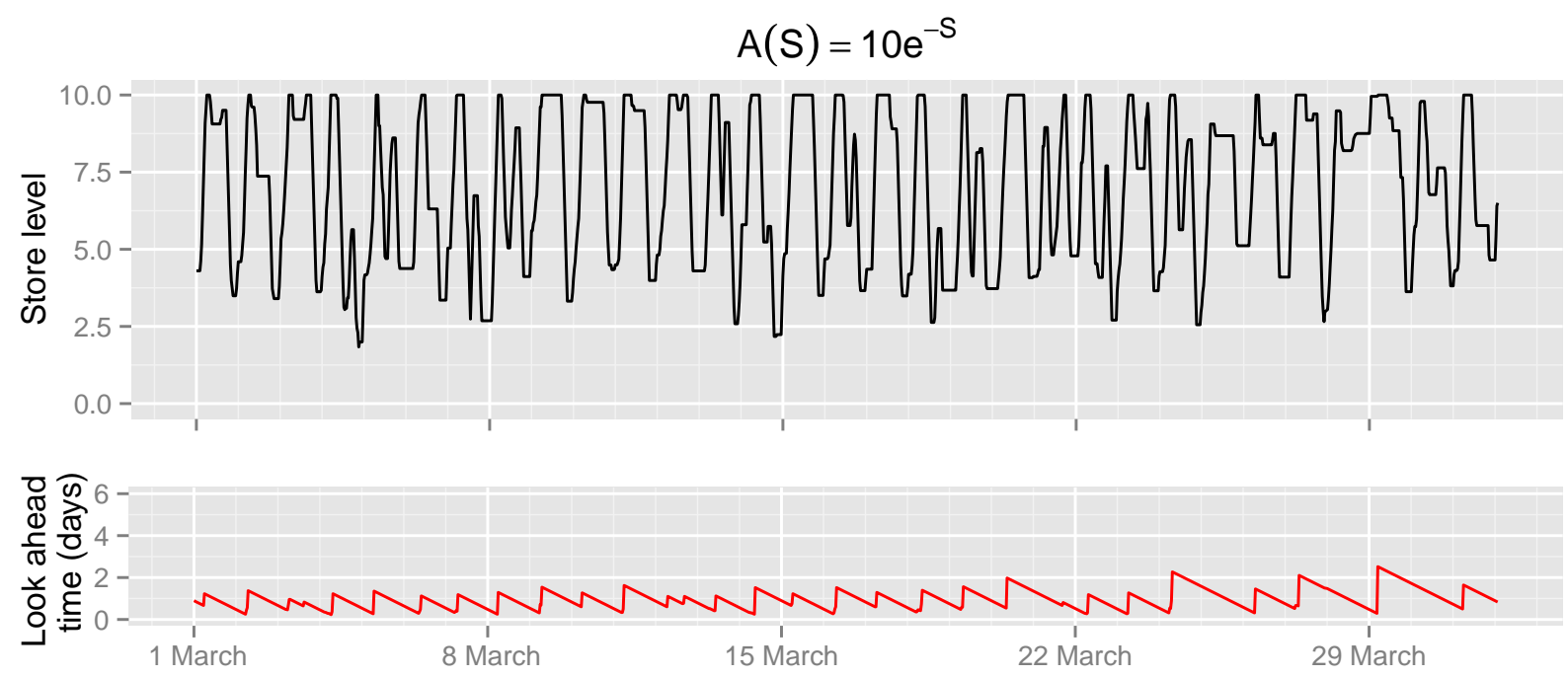

Figure 2: Store level and time horizon throughout March 2011 for the example with $A_{t}(s)=a e^{-\kappa s}$. The top panels correspond to $a=0$, the central panels to $a=1, \kappa=1$, and the bottom panels to $a=10, \kappa=1$. 
$A(S)=0$
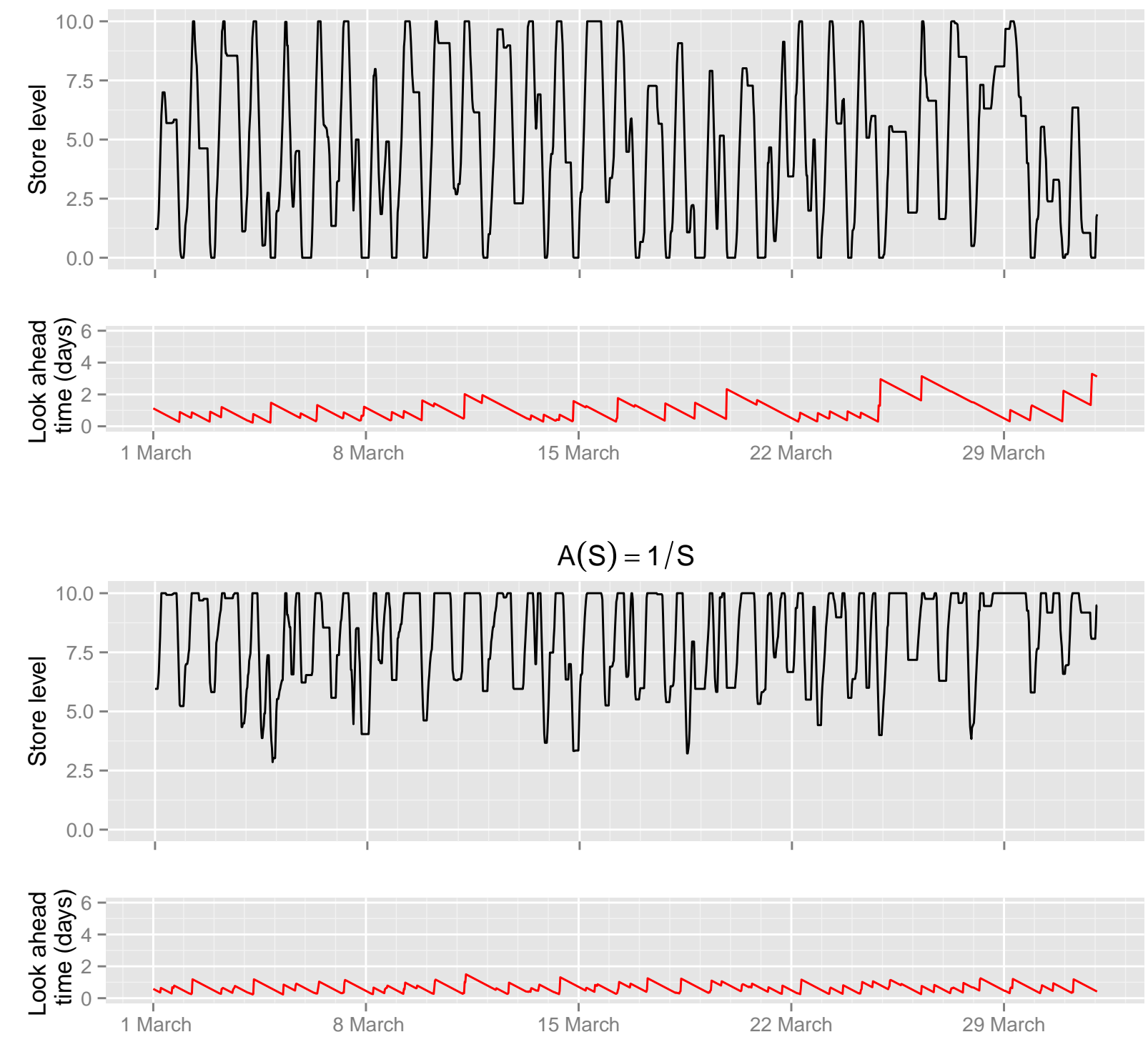

Figure 3: Store level and time horizon throughout March 2011 for the example with $A_{t}(s)=b / s$. The upper panels correspond to $A_{t}(s)=0$ and the lower panels to $A(t)=1 / s$.

\section{Acknowledgements}

The authors acknowledge the support of the Engineering and Physical Sciences Research Council for the programme (EPSRC grant EP/I017054/1) under which the present research is carried out. They also acknowledge the benefit of very helpful discussions with numerous colleagues, notably: Janusz Bialek, Chris Dent, Lisa Flatley, Richard Gibbens, Frank Kelly and Phil Taylor. 


\section{References}

[1] A. S. Cahn, "The warehouse problem," Bulletin of the American Mathematical Society, vol. 54, no. 11, pp. 1073-1073, 1948.

[2] R. Bellman, "On the theory of dynamic programming - a warehousing problem," Management Science, vol. 2, no. 3, pp. 272-275, 1956.

[3] S. E. Dreyfus, "An analytic solution of the warehouse problem," Management Science, vol. 4, no. 1, pp. 99-104, 1957.

[4] N. Secomandi, "Optimal commodity trading with a capacitated storage asset," Management Science, vol. 56, no. 3, pp. 449-467, 2010.

[5] J. R. Cruise, L. C. Flatley, R. J. Gibbens, and S. Zachary, "Optimal control of storage incorporating market impact and with energy applications." http://arxiv.org/abs/1406.3653, 2014.

[6] I. Koutsopoulos, V. Hatzi, and L. Tassiulas, "Optimal energy storage control policies for the smart power grid," in Proc. IEEE SmartGridComm, pp. 475-480, 2011.

[7] D. Pudjianto, M. Aunedi, P. Djapic, and G. Strbac, "Whole-systems assessment of the value of energy storage in low-carbon electricity systems," IEEE Transactions on Smart Grid, vol. 5, pp. 1098-1109, 2014.

[8] R. Sioshansi, P. Denholm, T. Jenkin, and J. Weiss, "Estimating the value of electricity storage in PJM: Arbitrage and some welfare effects," Energy Economics, vol. 31, pp. 269-277, Mar. 2009.

[9] P. van de Ven, N. Hegde, L. Massoulié, and T. Salonidis, "Optimal control of end-user energy storage," IEEE Transactions on Smart Grid, vol. 4, pp. 789-797, 2013.

[10] R. Walawalkar, J. Apt, and R. Mancini, "Economics of electric energy storage for energy arbitrage and regulation in New York," Energy Policy, vol. 35, pp. 2558-2568, Apr. 2007.

[11] J. C. Williams and B. D. Wright, Storage and Commodity Markets. Cambridge University Press, 2005.

[12] F. Teng, J. Miles, A. Thomson, G. Strbac, N. Brandon, and D. Pudjianto, "Potential value of energy storage in the UK electricity system," Proceedings of the ICE - Energy, vol. 168, pp. 107-117, May 2015.

[13] E. D. Castronuovo and J. A. P. Lopes, "Optimal operation and hydro storage sizing of a windhydro power plant," International Journal of Electrical Power 83 Energy Systems, vol. 26, pp. 771-778, Dec. 2004.

[14] P. Denholm and R. Sioshansi, "The value of compressed air energy storage with wind in transmission-constrained electric power systems," Energy Policy, vol. 37, pp. 31493158, Aug. 2009.

[15] M. Korpaas, A. T. Holen, and R. Hildrum, "Operation and sizing of energy storage for wind power plants in a market system," International Journal of Electrical Power E Energy Systems, vol. 25, pp. 599-606, Oct. 2003. 
[16] P. Harsha and M. Dahleh, "Optimal management and sizing of energy storage under dynamic pricing for the efficient integration of renewable Energy," IEEE Transactions on Power Systems, vol. 30, pp. 1164-1181, May 2015.

[17] N. Richmond, P. Jacko, and A. M. Makowski, "Optimal planning of slow-ramping power production in energy systems with renewables forecasts and limited storage," in 2014 International Conference on Probabilistic Methods Applied to Power Systems (PMAPS), pp. 1-6, IEEE, July 2014.

[18] A. Tuohy and M. O'Malley, "Impact of pumped storage on power systems with increasing wind penetration," in 2009 IEEE Power $\&$ Energy Society General Meeting, pp. 1-8, IEEE, July 2009.

[19] O. Megel, J. L. Mathieu, and G. Andersson, "Maximizing the potential of energy storage to provide fast frequency control," in IEEE PES ISGT Europe 2013, pp. 1-5, IEEE, Oct. 2013.

[20] A. Oudalov, D. Chartouni, and C. Ohler, "Optimizing a battery energy storage system for primary frequency control," IEEE Transactions on Power Systems, vol. 22, pp. 1259-1266, Aug. 2007.

[21] A. Bernstein, L. Reyes-Chamorro, J.-Y. Le Boudec, and M. Paolone, "A composable method for real-time control of active distribution networks with explicit power setpoints. Part I: Framework," Electric Power Systems Research, vol. 125, pp. 254 - 264, 2015 .

[22] Y. Huang, S. Mao, and R. Nelms, "Adaptive electricity scheduling in microgrids," in Proc. IEEE INFOCOM, Turin, Italy, 2013.

[23] J. P. Barton and D. G. Infield, "Energy storage and its use with intermittent renewable energy," IEEE Transactions on Energy Conversion, vol. 19, no. 2, pp. 441-448, 2004.

[24] A. Bejan, R. Gibbens, and F. Kelly, "Statistical aspects of storage systems modelling in energy networks," in 46th Annual Conference on Information Sciences and Systems (invited session on Optimization of Communication Networks), Princeton University, USA, 2012.

[25] N. G. Gast, D.-C. Tomozei, and J.-Y. Le Boudec, "Optimal storage policies with wind forecast uncertainties," in Greenmetrics 2012, Imperial College, London, UK, 2012.

[26] M. Arnold and G. Andersson, "Model predictive control of energy storage including uncertain forecasts," Proceedings of the 17th Power Systems Computation Conference, 2011.

[27] S. D. Howell, P. W. Duck, A. Hazel, P. V. Johnson, H. Pinto, G. Strbac, N. Proudlove, and M. Black, "A partial differential equation system for modelling stochastic storage in physical systems with applications to wind power generation.," IMA Journal of Management Mathematics, vol. 22, pp. 231-252, 2011.

[28] J. P. Barton and D. G. Infield, "A probabilistic method for calculating the usefulness of a store with finite energy capacity for smoothing electricity generation from wind and solar power," Journal of Power Sources, vol. 162, pp. 943-948, 2006. 
[29] "Electricity balancing services." National Audit Office Briefing, May 2014.

http://www.nao.org.uk/wp-content/uploads/2014/05/

Electricity-Balancing-Services.pdf.

[30] "The electricity trading arrangements." ELEXON.

https://www.elexon.co.uk/wp-content/uploads/2013/11/beginners_guide_ to_trading_arrangements_v4.0_cgi.pdf.

[31] R. Billinton and R. N. Allan, Reliability Evaluation of Power Systems. Springer, 2 ed., 1996.

[32] S. Boyd and L. Vandenberghe, Convex Optimization. Cambridge University Press, 2004.

[33] P. Whittle, Optimization Under Constraints: Theory and Applications of Nonlinear Programming. Wiley, 1971.

[34] "Dinorwig power station."

http://www.electricmountain.co.uk/Dinorwig-Power-Station. 